\title{
Choroidal Hemangioma Associated with Hepatic Hemangioma: A Case Report and Literature Review
}

\author{
Ana Boned-Murillo ${ }^{a}$ Maria Dolores Diaz-Barreda ${ }^{a}$ \\ Ana Honrubia-Grijalbo ${ }^{a}$ b Olivia Esteban-Floria ${ }^{a}$ \\ Francisco Javier Ascaso Puyueloa, b \\ aDepartment of Ophthalmology, Lozano Blesa University Clinic Hospital, Zaragoza, Spain; \\ ${ }^{b}$ Aragon Health Research Institute (IIS Aragon), Zaragoza, Spain
}

\section{Keywords}

Circumscribed choroidal hemangioma · Hepatic hemangioma · GNAQ · GNA11

\begin{abstract}
Circumscribed choroidal hemangioma $(\mathrm{CCH})$ is a rare congenital ocular tumor type that is usually benign and asymptomatic. $\mathrm{CCH}$ has only been reported once previously in the literature in association with a visceral neoplasm. Here, we present a newly described association between $\mathrm{CCH}$ and hepatic hemangioma $(\mathrm{HH})$. We report the case of a 57-year-old woman diagnosed with asymptomatic $\mathrm{HHs}$ who presented with a 2- to 3-month history of central scotoma and blurred vision in her left eye. Assessment identified an orange-red elevated lesion with a central serous retinal detachment and subretinal edema. Complementary tests suggested a $\mathrm{CCH}$. To our knowledge, $\mathrm{CCH}$ has only been reported once previously in association with a visceral neoplasm, making this newly described association between $\mathrm{CCH}$ and $\mathrm{HH}$ of clinical relevance. Ophthalmologists should be aware of the possible association between $\mathrm{CCH}$ and other visceral tumors as this association offers an opportunity for the early detection of this pathology.
\end{abstract}

\section{Introduction}

Choroidal hemangioma $(\mathrm{CH})$ is classified as circumscribed $\mathrm{CH}(\mathrm{CCH})$ when not presented with extraocular associations $[1,2]$. CCH is asymptomatic until adulthood, where it presents as blurred vision secondary to exudative retinal detachment or hyperopic shift [2-4]. Histological classification comprises 3 types, with stratification according to the size of the affected vessels [1,5]. Furthermore, oncogenic alterations of the $G N A Q$ and GNA11 genes [6] have been 
detected in endothelial cells of capillary malformations such as $\mathrm{CCH}$ [7]. Hepatic hemangiomas (HHs) are "incidentalomas," clusters of blood-filled cavities lined by endothelial cells and fed by the hepatic artery [8]; they are generally asymptomatic and stationary in size. The association between $\mathrm{CCH}$ and visceral neoplasms has only been described once, with the case of a CCH associated with infantile hepatic hemangioendotheliomatosis (IHE) [9]. Here, we report a case of a CCH of the left eye in a woman with a history of 2 asymptomatic HHs.

\section{Case Presentation}

We present the case of a 57-year-old Caucasian woman complaining of a 2- to 3-month history of central scotoma and blurred vision at distance in her left eye. She presented no allergies or known toxic habits and an unremarkable ocular history. Her medical history included hepatitis B, arterial hypertension, and hyperlipidemia; additionally, 2 hyperechogenic images of 8.6 and $16.8 \mathrm{~mm}$ in diameter at the right hepatic lobe were reported, homogeneous with smooth contours, classified as HH (Fig. 1). At her first visit, her best-corrected visual acuity (BCVA) was 20/20 and 20/63 in the right and left eyes, respectively. Confrontation fields were full, extraocular muscles had a full range of motion, and her pupils were equally reactive. Slit-lamp biomicroscopy examination was normal, and Goldmann applanation intraocular pressure was $16 \mathrm{~mm} \mathrm{Hg}$ in both eyes. Dilated fundus examination of the left eye revealed an orange-red elevated lesion close to the optic nerve (Fig. 2). Optical coherence tomography revealed an increased subfoveal retinal thickness $(661 \mu \mathrm{m})$ with a central serous retinal detachment and an underlying choroidal mass (Fig. 3, 4). Fundus photography, magnetic resonance imaging (Fig. 5), and B-scan ultrasonography (Fig. 6) showed a $5 \times 3 \times 4$-mm solid choroidal mass medial to the optic nerve head with vessels that continued with retinal vascularization on B-scan and high internal reflectivity on A-scan. Fluorescein angiography demonstrated hyperfluorescence in all stages (Fig. 7). The patient was diagnosed with $\mathrm{CCH}$.

After 2 weeks, and without any intervention, BCVA was 20/32 in her left eye, the volume of subretinal fluid was reduced, and the lesion showed no signs of change (Fig. 8). Dilated fundus examination and optical coherence tomography were performed periodically, revealing a progressive reduction of the volume of subretinal fluid. After 9 months, serous retinal detachment was resolved (Fig. 4), and BCVA 20/20 and 20/25 in the right and left eyes, respectively. During the following 4 years, the patient remained asymptomatic and the lesion remained stable (Fig. 3).

Fig. 1. HH ultrasonography: abdominal Bscan ultrasonography showing a normal size homogeneous liver with smooth contours, with 2 hyperechogenic images of 8.6 and $16.8 \mathrm{~mm}$ in diameter at the right liver lobe, which were classified as HHs. $\mathrm{HH}$, hepatic hemangioma.

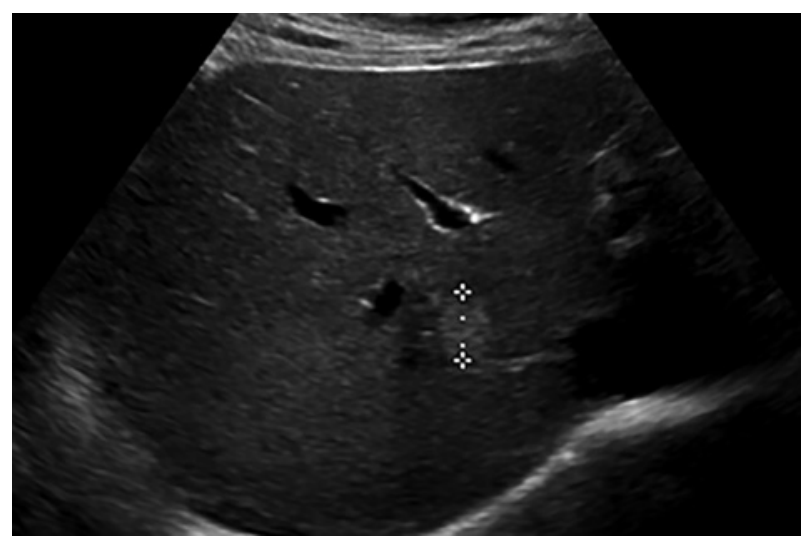




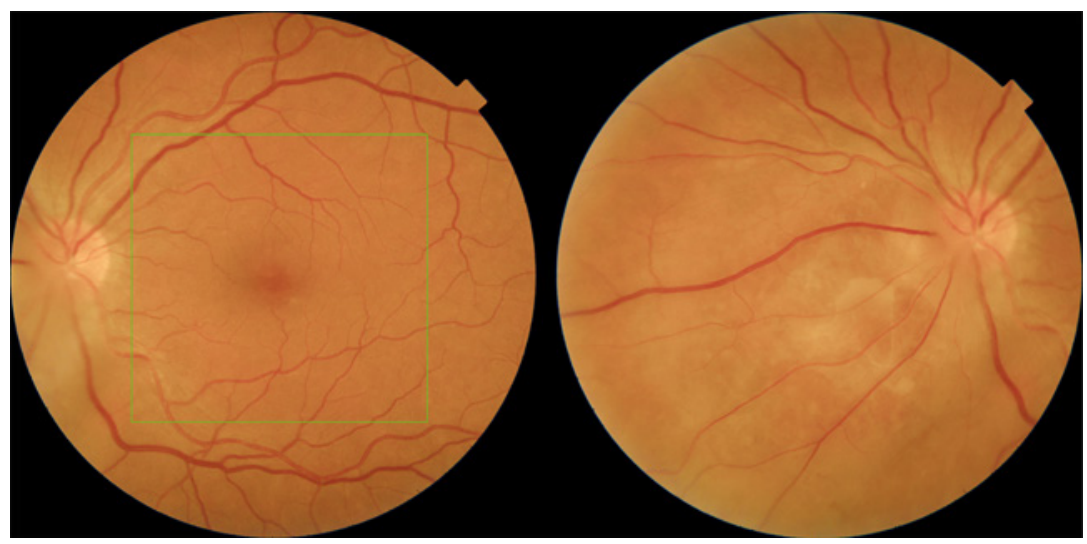

Fig. 2. $\mathrm{CCH}$ fundoscopic: dilated fundus examination of the left eye revealing an orange-red elevated lesion medial to the optic nerve. The appearance of the macula was unaltered. $\mathrm{CCH}$, circumscribed choroidal hemangioma.
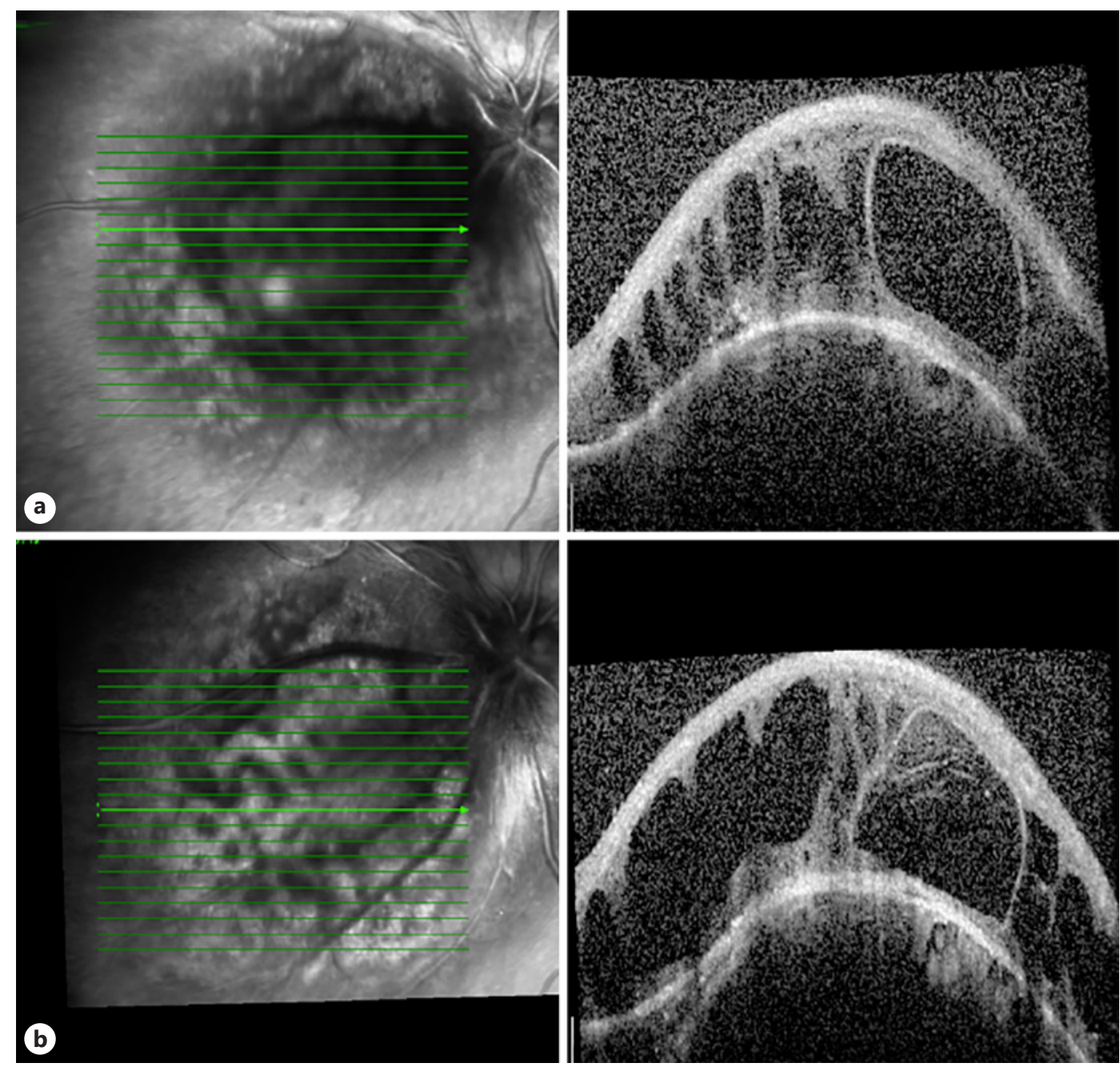

Fig. 3. СCH OCT progression: OCT revealing an underlying large choroidal mass with intraretinal fluid (a) which remains stable after 4 years (b). CCH, circumscribed choroidal hemangioma; OCT, optical coherence tomography. 

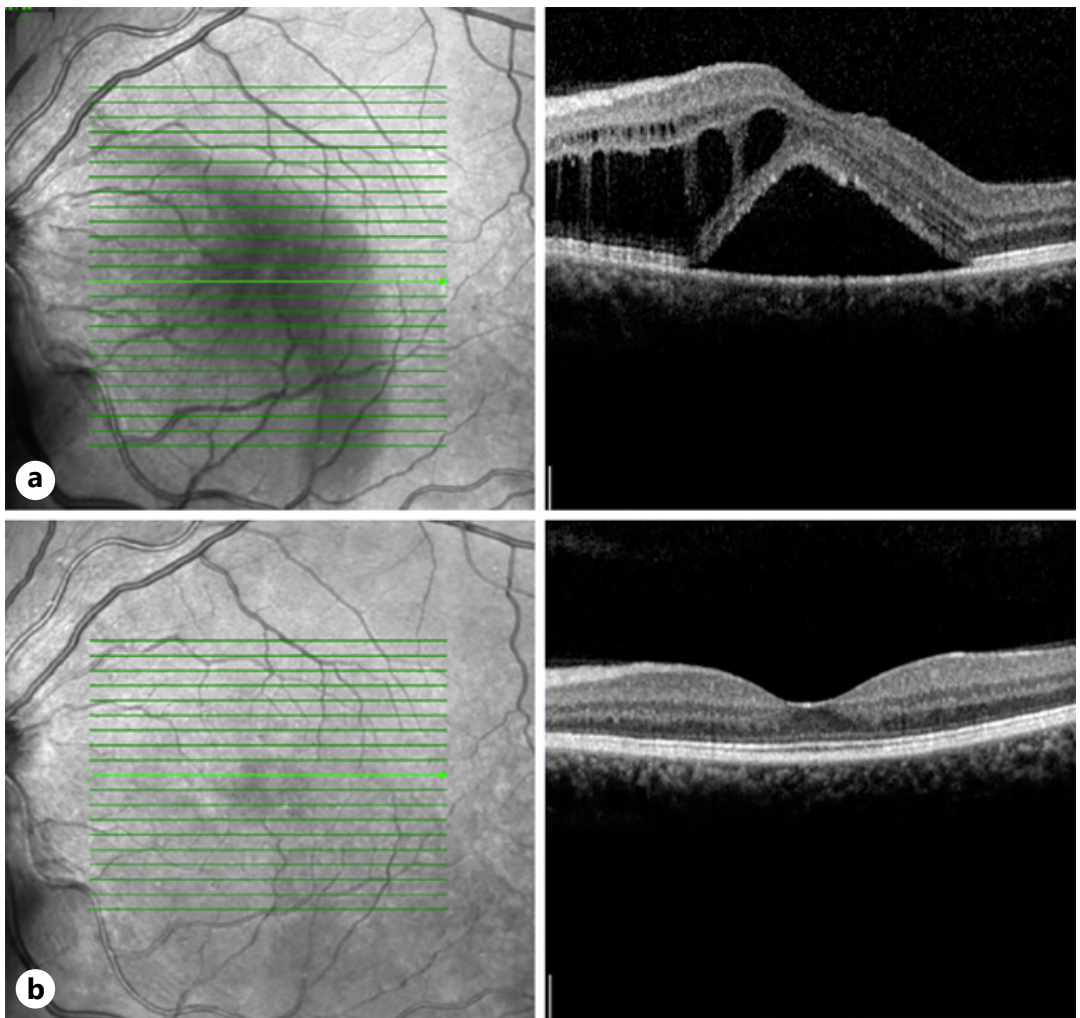

Fig. 4. Macula OCT progression: a OCT revealing an increased subfoveal retinal thickness (661 $\mu \mathrm{m})$ with a central serous retinal detachment, with intraretinal and subretinal fluid. Some hyperreflective dots bordering the serous retinal detachment was observed suggestive of a possible chronicity of the edema. $\mathbf{b}$ After 9 months, an improvement in subretinal fluid and the resolution of serous retinal detachment is observed. OCT, optical coherence tomography.

Fig. 5. CCH MRI: MRI revealing a hyperintense mass in T1-weighted images and isointense to the vitreous in T2-weighted images, with a marked enhancement on gadolinium administration. $\mathrm{CCH}$, circumscribed choroidal hemangioma.

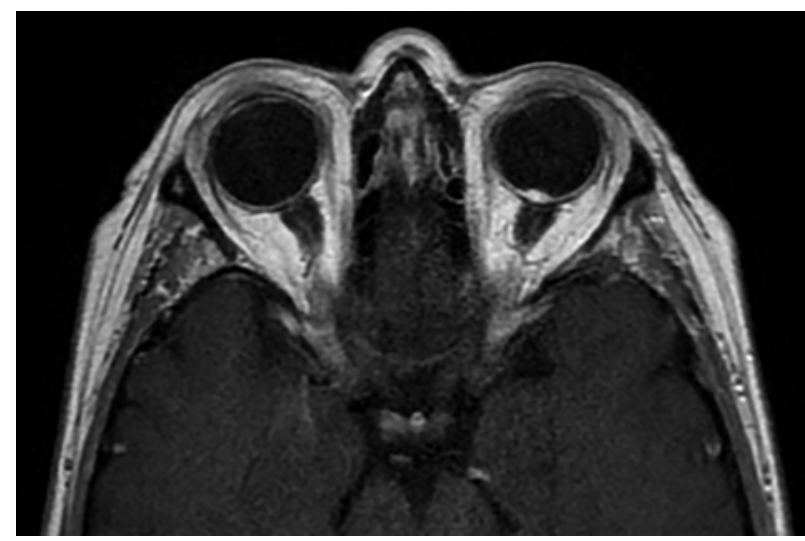

\section{Discussion and Conclusions}

$\mathrm{CCH}$ is a type of benign vascular hamartoma composed of an irregular congestive and dilated network of choroidal vessels separated by a thin intervascular septum; there is no choriocapillaris involvement, and, unlike other "true hemangiomas," they do not show cellular proliferation and present slow to no progression in size over time $[3,10]$. Histological 

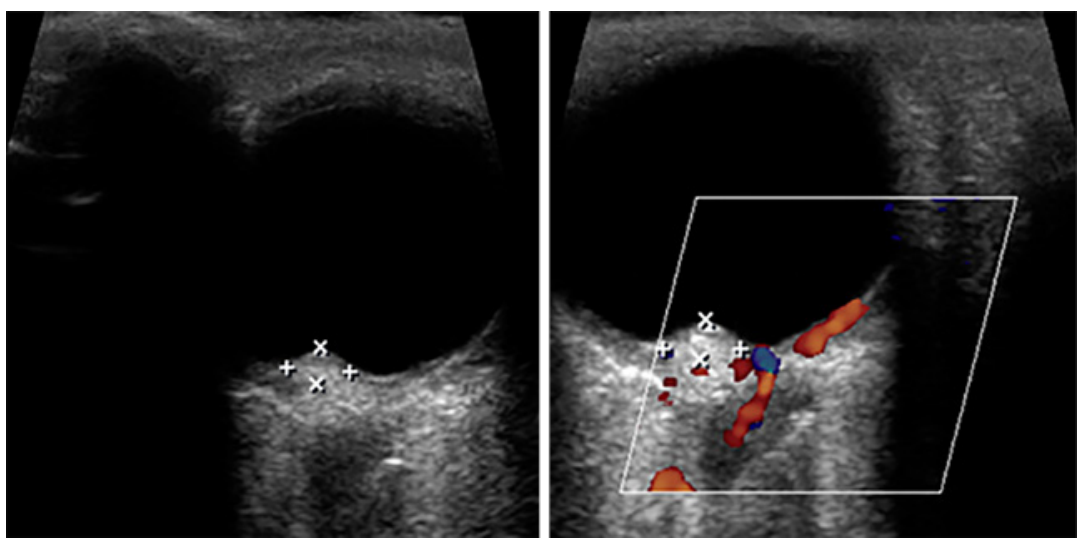

Fig. 6. CCH ultrasonography: A- and B-scan ultrasonography showing high surface reflectivity with high to medium internal reflectivity and exudative retinal detachment. $\mathrm{CCH}$, circumscribed choroidal hemangioma.

Fig. 7. CCH fluorescein angiography: fluorescein angiography showing early hyperfluorescence with an intense leakage. The inferior extent of the leakage is well-appreciated in the form of retinal pigment epithelial tracts. $\mathrm{CCH}$, circumscribed choroidal hemangioma.

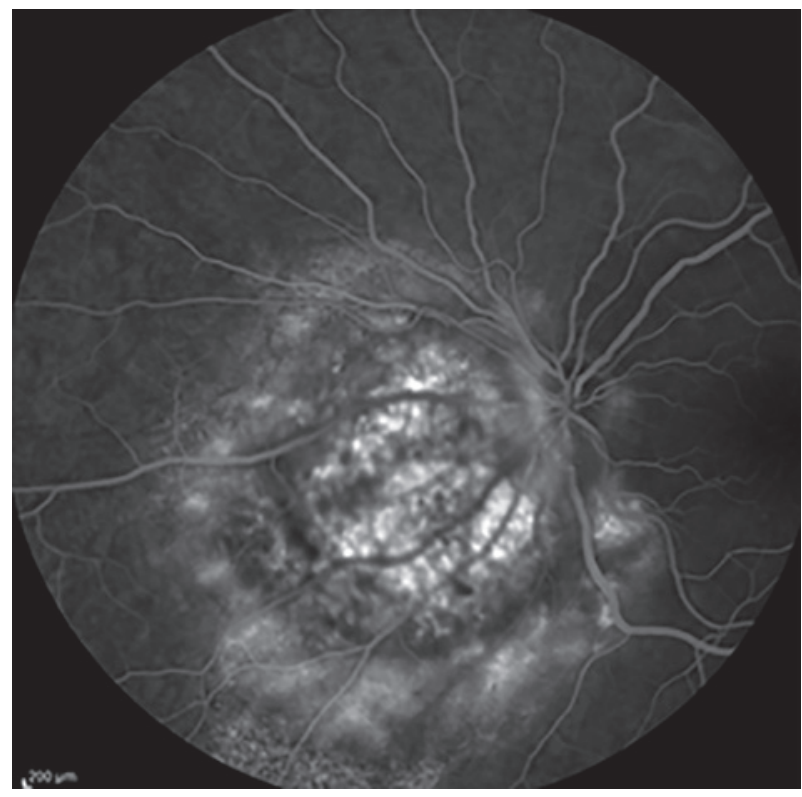

classification is as follows: (1) capillary type, which is rare and composed of small blood vessels with inconspicuous endothelial cells separated by loose connective tissue; (2) cavernous type, which is more common and consists of large, thin-walled, blood-filled vascular channels lined by a flat endothelium and separated by a thin intervascular septum; and (3) mixed type, which is a mixture of both types $[1,5]$.

$\mathrm{CCH}$ management is based on the location and size characteristics, and ocular symptoms, with chronic subretinal fluid and chronic macular edema being the most common causes for decreased vision. In this case, as in asymptomatic hemangiomas, observation can be considered, but a possible neovascular glaucoma may be developed [5].

Generally, no systemic workup is needed as there are no associated systemic complications to $\mathrm{CCH}$. Association with other visceral hemangiomas is very rare. To our knowledge, this is the second noted association of a CCH with a visceral neoplasm. The first was reported by Shaikh et al. [11] as a CCH associated with IHE, a condition characterized by congenital lesions noted either at birth or during the first 6 months of life, and often accompanied by the 

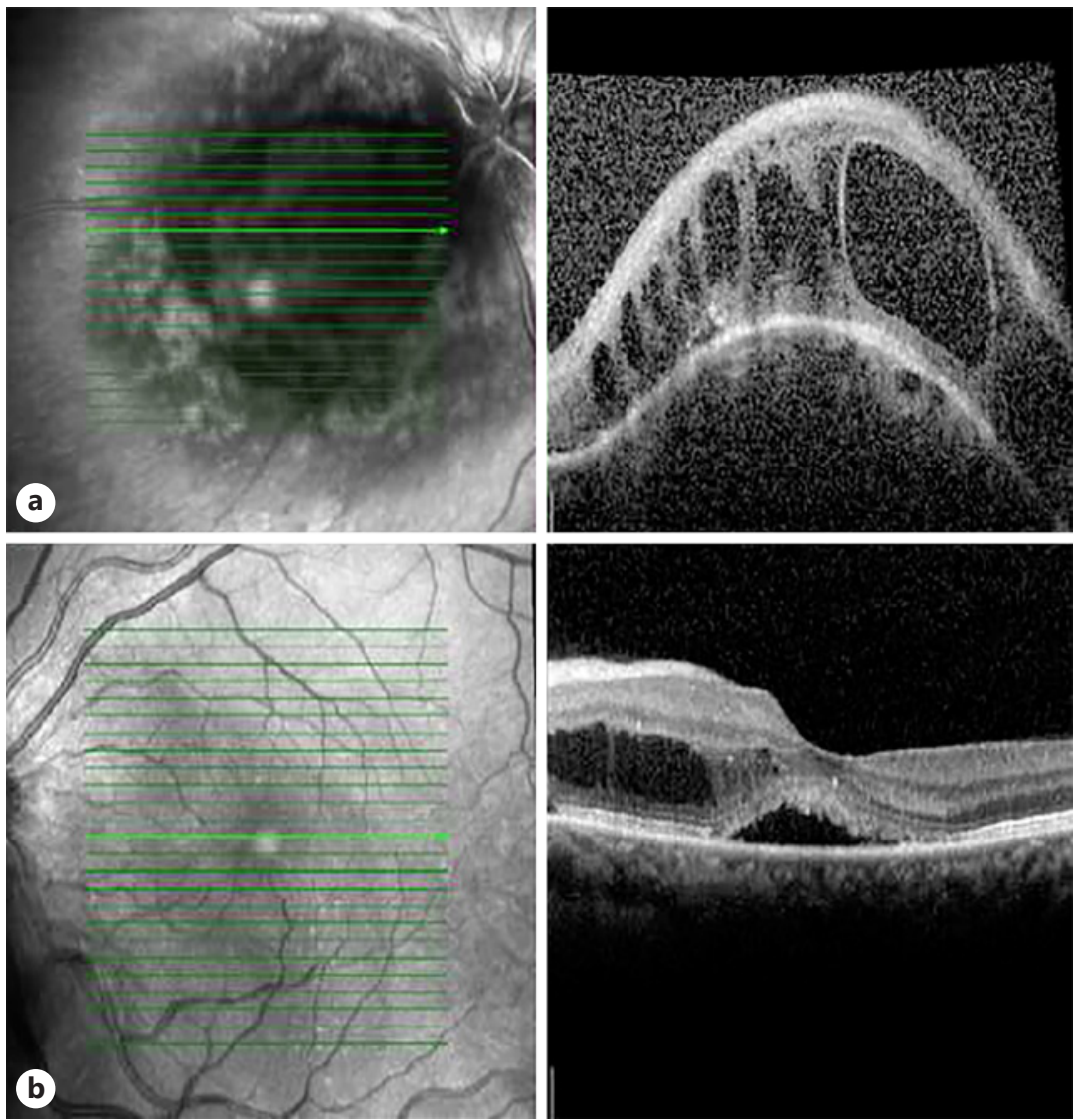

Fig. 8. OCT after 2 weeks without intervention, the lesion showed no signs of change (a), but the volume of subretinal fluid was reduced and BCVA improved (b). BCVA, best-corrected visual acuity; OCT, optical coherence tomography.

classic symptomatic triad of hepatomegaly, congestive heart failure, and hemangiomas of the skin [9].

Histologically, similar to CCH, IHE is a benign hamartoma composed of anastomosing vascular channels lined with endothelial cells $[9,11,12]$. Some authors support the theory of a common pathology for $\mathrm{CCH}$ and hepatic and cutaneous hemangioendotheliomas, likely related to persistent arteriovenous shunts that develop during the embryogenesis of the choroidal vasculature [9]. While HH may remain asymptomatic until adulthood, CCH may present as an associated vascular visceral abnormality in its congenital form $[9,11,12]$. Since infantile hepatic hemangioendotheliomatosis and cutaneous hemangioendotheliomas show a high incidence of spontaneous regression, treatment is only recommended when associated conditions present a high risk of morbidity; this treatment approach is also applied to $\mathrm{CCH}$.

CCH and HH association may also be related to the mutation of GNAQ or GNA11 p.Q209, which keeps the alpha subunit of a heterotrimeric G protein in its active guanosine triphosphate-bound state [6]. Different mutational profiles of GNAQ and GNA11 may be presented, establishing 4 clusters $[7,13,14]$. The second and third main clusters are predominated by alterations, resulting in GNAQ or GNA11 p.Q209 mutation status-positive disease characterized by continuous cell proliferation and which usually presents during adulthood in the form of a vascular tumor (such as $\mathrm{CCH}$, hepatic small-vessel neoplasia, congenital hemangioma, and $\mathrm{HH}$ ) [13] or a melanocytic tumor [6]. 
Additional studies are needed to further investigate the association between $\mathrm{CCH}$ and $\mathrm{HH}$ as the literature related to this topic is scarce. Moreover, the literature places the $\mathrm{HH}$ incidence at $0.4-20 \%$ of the total population and over $7 \%$ at necropsy [8]. The fact that HHs are "incidentalomas" should be considered at diagnosis as the presence of an HH could be an incidental finding in a patient with $\mathrm{CCH}$, and there may not be an association.

In conclusion, we present a newly described association between $\mathrm{CCH}$ and $\mathrm{HH}, \mathrm{CCH}$ having previously only been reported in association with IHE. Ophthalmologists should be aware of the possible association between $\mathrm{CCH}$ and visceral tumors as this association offers an opportunity for the early detection of this pathology.

\section{Statement of Ethics}

Written informed consent was obtained from the patient for publication of this case report and any accompanying images and videos. A copy of the written consent is available for review by the editor of this Journal.

\section{Conflict of Interest Statement}

The authors declare that they have no competing interests.

\section{Funding Sources}

There is no funding body in the design of the study and collection, analysis, and interpretation of data and in writing the manuscript.

\section{Author Contributions}

H.A. and A.J. analyzed and interpreted the patient data regarding the ophthalmic disease. E.O. performed the ocular examination of the eye. D.M.D., B.A., and A.J. were major contributors in writing the manuscript. D.M.D. has contributed substantially to the draft and revision of the work. All authors read and approved the final manuscript.

\section{Availability of Data and Material}

The datasets generated and/or analysed during the current study are available in the PubMed repository, https://www.ncbi.nlm.nih.gov/pubmed/.

\section{References}

1 Shanmugam PM, Ramanjulu R. Vascular tumors of the choroid and retina. Indian J Ophthalmol. 2015;63(2): 133-40.

2 Spanish Vitreo-Retinal Society (SERV). Pigmented fundus lesions guide "SERV guidelines for clinical practice". Arch Soc Esp Oftalmol. 2012. Disponible at: www.serv.es.

3 Berry M, Lucas LJ. Circumscribed choroidal hemangioma: a case report and literature review. J Optom. 2017; 10(2):79-83.

\section{Karger's}


4 Singh AD, Kaiser PK, Sears JE. Choroidal hemangioma. Ophthalmol Clin North Am. 2005;18(1):151-ix.

5 Mashayekhi A, Shields CL. Circumscribed choroidal hemangioma. Curr Opin Ophthalmol. 2003;14(3):142-9.

6 Le Guin CHD, Metz KA, Kreis SH, Bechrakis NE, Bornfeld N, Zeschnigk M, et al. Gnaq q209r mutations are highly specific for circumscribed choroidal hemangioma. Cancers. 2019;11(7):1031.

7 Couto JA, Huang L, Vivero MP, Kamitaki N, Maclellan RA, Mulliken JB, et al. Endothelial cells from capillary malformations are enriched for somatic GNAQ mutations. Plast Reconstr Surg. 2016;137(1):77e-82e.

8 Bajenaru N, Balaban V, Săvulescu F, Campeanu I, Patrascu T. Hepatic hemangioma: review. J Med Life. 2015;8 Spec Issue:4-11.

9 Zimmermann A. Infantile hepatic hemangioma/hemangioendothelioma. Tumors and tumor-like lesions of the hepatobiliary tract. Switzerland: Springer International Publishing; 2016. p. 937-57.

10 Karimi S, Nourinia R, Mashayekhi A. Circumscribed choroidal hemangioma. J Ophthalmic Vis Res. 2015;10(3): $320-8$.

11 Shaikh S, Sanislo S, O'Brien JM, Alcorn D. Congenital circumscribed choroidal haemangioma associated with infantile hepatic haemangioendotheliomatosis. Br J Ophthalmol. 2001;85(5):626-5.

12 Shields CL, Manalac J, Das C, Saktanasate J, Shields JA. Review of spectral domain enhanced depth imaging optical coherence tomography of tumors of the choroid. Indian J Ophthalmol. 2015;63(2):117-21.

13 Ayturk UM, Couto JA, Hann S, Mulliken JB, Williams KL, Huang AY, et al. Somatic activating mutations in GNAQ and GNA11 are associated with congenital hemangioma. Am J Hum Genet. 2016;98(4):789-95.

14 Francis JH, Milman T, Grossniklaus H, Albert D, Folberg R, Levitin G, et al. GNAQ mutations in diffuse and solitary choroidal hemangiomas. Ophthalmology. 2019;126(5):759-63. 